\title{
UNIQUE ELECTRON POLARIMETER COMPARISON AND SPIN-BASED ENERGY MEASUREMENT
}

\author{
J. Grames* ${ }^{\dagger}$, TJNAF, Newport News, VA 23606, USA
}

\section{Abstract}

A careful intercomparison of the relative analyzing power of five electron beam polarimeters was performed with the CEBAF accelerator at the Thomas Jefferson National Accelerator Facility (JLab) during a dedicated two day machine development period. This is the first time such a high precision comparison between polarimeters of the Mott, Compton, and Møller type has been made. A Wienstyle spin manipulator at the injector was used to vary the spin orientation of the electron beam. A series of polarization measurements as a function of spin orientation, determines the relative analyzing power between the five polarimeters. More importantly, the high statistical precision of the measurements reveal the relative differences between the polarimeters which are systematic in nature and may ultimately help realize high precision absolute electron polarimetry. In addition, a comparison of the value of the injector spin angle that provides precise longitudinal beam polarization at each experimental hall leads to an independent and potentially high precision measurement with relative uncertainty (better than $10^{-4}$ ) of the final electron beam energy.

\section{INTRODUCTION}

Electron beam polarimetry is the technique of separating scattered particles for detection using some physical interaction between the polarization of an electron beam $(\mathrm{P})$ and the total analyzing power of a polarimeter's target $\left(A_{t o t}\right)$. The target is itself polarized in many polarimeter designs and $A_{t o t}$ is then proportional to the product of the target polarization and the analyzing power of the interaction. In each case the scattering process results in a measured experimental asymmetry $(\epsilon)$,

$$
\epsilon=A_{t o t} \cdot P \text {. }
$$

The kinematics and design of each polarimeter determine which components of the total beam polarization are measured. A total of five electron beam polarimeters of three types (Mott, Compton, Møller) are used at JLab. These are summarized in Tab. 1. The main purpose of this experiment is to compare the relative analyzing power of the electron polarimeters. A parallel result of the experiment also provides precise measurement of the final beam energy using two independent spin precession techniques.

\footnotetext{
*grames@jlab.org

$\dagger$ This work was supported by the U.S. DOE Contract No. DE-AC0584-ER40150.
}

Table 1: Five JLab electron beam polarimeters.

\begin{tabular}{|l|c|r|l|}
\hline Type & Reaction & $\mathbf{I}_{\text {ave }}$ & Target \\
\hline Injector Mott & $\vec{e}+Z A$ & $5 \mu \mathrm{A}$ & $\mathrm{Au}$ \\
Hall A Compton & $\vec{e}+\vec{\gamma}$ & $100 \mu \mathrm{A}$ & Photon \\
Hall A Møller & $\vec{e}+\vec{e}$ & $2 \mu \mathrm{A}$ & Fe-alloy \\
Hall B Møller & $\vec{e}+\vec{e}$ & $5 \mathrm{nA}$ & $\mathrm{Fe}-$ alloy \\
Hall C Møller & $\vec{e}+\vec{e}$ & $2.5 \mu \mathrm{A}$ & $\mathrm{Fe}$ \\
\hline
\end{tabular}

\section{THE EXPERIMENT}

The experimental asymmetry measured at each polarimeter is proportional to the projection of the total beam polarization along some analyzing component of the polarimeter. The capability for simultaneous beam delivery to multiple end-station polarimeters at JLab has the advantage that each measurement is of the same uniquely polarized beam. However, the measurable component of the beam polarization with respect to each polarimeter is generally not equal due to precession of the electron spin.

A solution is to perform the polarimetry in a way which does not rely upon only one measurement of a single component of the beam polarization. This is accomplished by adjusting the orientation of the beam polarization using a spin rotator at a location common to all delivered beams. The measured components of the beam polarization can then be plotted against this orientation parameter. A fit of the data yields the experimental asymmetry and the spin rotation angle which results in the maximum longitudinal polarization at each polarimeter.

\subsection{The Wien Filter}

The spin rotator used to accomplish this is a Wien filter which consists of crossed electric $(\vec{E})$ and magnetic $(\vec{B})$ fields transverse to the particle motion $(\vec{\beta})$ and each other as shown in Fig. 1. The usefulness of the Wien filter is that

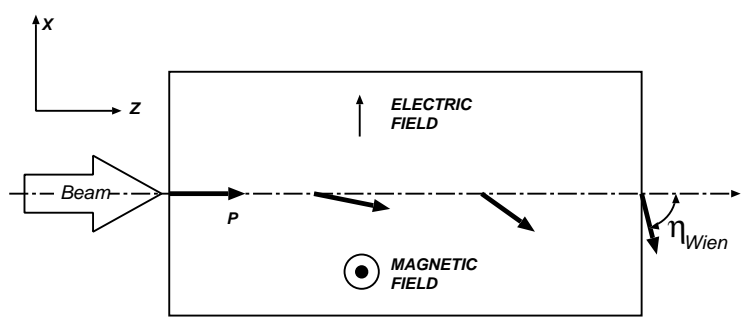

Figure 1: Diagram of Wien filter indicating the rotation of the beam polarization relative to the beam direction $\left(\eta_{W i e n}\right)$ in crossed magnetic and electric fields $\left(\beta=\frac{E}{B}\right)$. 
the polarization of a beam passing through the device can be rotated by an angle with respect to the beam momentum $\left(\eta_{\text {Wien }}\right)$ without deflecting the outgoing orbit. This is accomplished by first setting the electric field of the Wien filter for the desired rotation and then applying a crossed magnetic field to set the net Lorentz force on the particle to zero,

$$
\vec{F}=q(\vec{E}+\vec{\beta} \times \vec{B})=0 .
$$

The second condition then requires that $\beta=\frac{E}{B}$. The precession is linear with the applied fields $\left(\eta_{W i e n}=\eta_{\vec{E}}+\eta_{\vec{B}}\right)$ and at the injector energy is dominated by the contribution from the electric field integral,

$$
\frac{\eta_{\vec{B}}}{\eta_{\vec{E}}}=\frac{-a \gamma}{\frac{g}{2 \gamma}-a \gamma}=-0.17 \%,
$$

where the Lorentz parameter $\gamma=1.196$ at $100 \mathrm{keV}, g$ is the electron gyromagnetic factor, and $a=\frac{g-2}{2}=1.159 \times$ $10^{-3}$.

A calibration of the power supplies used to set the Wien filter electric field was performed to determine the applied differential voltage as a function of DAC setpoint. A second-order polynominal fit to account for bias voltage, gain, and linearity in response was made,

$$
V_{W i e n}= \pm\left(p_{0}+p_{1} \cdot S_{d a c}+p_{2} \cdot S_{d a c}^{2}\right),
$$

as reported in Fig. 2. The sign of the expression depends upon the state of a high voltage switchbox.
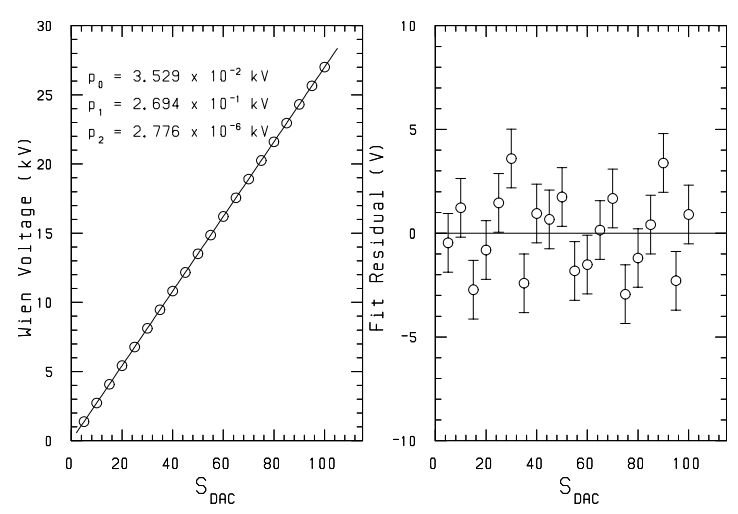

Figure 2: Differential Wien voltage with fit (left) and residuals (right) shown versus DAC setpoint $\left(S_{D A C}\right)$.

\subsection{Collecting the Data}

Polarimeter data was collected at 12 Wien angles spanning $220^{\circ}$. The Mott and Moller polarimeters used a DC laser generated electron beam that was RF chopped in the injector making three interleaved $499 \mathrm{MHz}$ beams. The beams were then either all directed to 1) the Mott polarimeter or 2) the three Møller polarimeters simultaneously (5pass) using RF separators in the beam switchyard. In a third configuration, the electron beam was RF laser generated $(1.497 \mathrm{GHz})$ at higher average current $(\sim 75 \mu \mathrm{A})$ for the Compton polarimeter. A simple schematic of the CEBAF accelerator and experimental halls is given in Fig. 3.

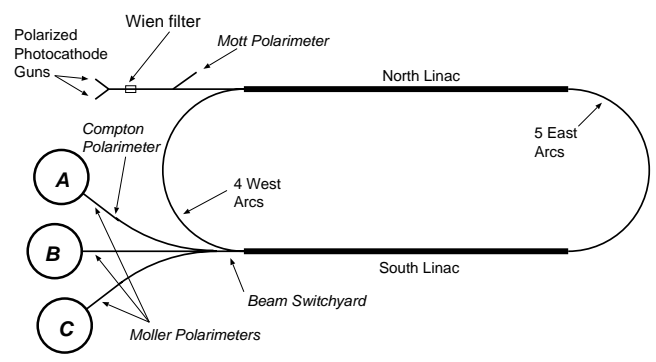

Figure 3: Schematic of the CEBAF 5-pass recirculating accelerator at JLab noting the Wien filter and five electron polarimeter locations.

\section{RESULTS}

The polarimeter data was analyzed by each respective polarimeter group and reported. The dependence of the measured polarization on the Wien angle is modeled using,

$$
P_{\text {measured }}=P_{0} \cos \left(\lambda \cdot V_{W i e n}+\Psi+\phi_{g}\right),
$$

where $P_{0}$ is the beam polarization amplitude and $\Psi$ is the net spin rotation between the Wien filter and polarimeter. The value $\phi_{g}=-0.01^{\circ}$ corrects for a dipole precession between the electron gun and Wien Filter. The constant coefficient $\lambda$, which relates the applied voltage to spin rotation, is a weighted average derived from the best-fit values for each polarimeter individually. The data and model fit
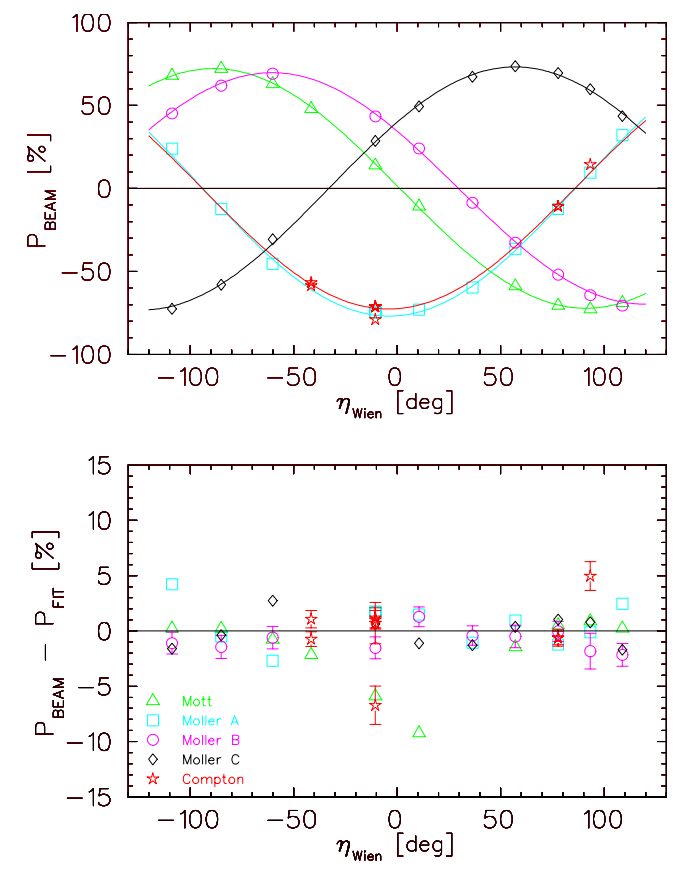

Figure 4: Polarimeter data and model fit (upper plot) with $\lambda=5.627^{\circ} / \mathrm{kV}$ and fit residuals (lower plot). Uncertainties shown are only statistical.

are shown in Fig. 4 while the numerical results of the fit are given in Tab. 2. Without considering the systematic un- 
Table 2: Amplitude and phase results using $\lambda=5.627^{\circ} / \mathrm{kV}$

\begin{tabular}{|l|r|r|}
\hline Type & Amplitude (\%) & Phase (deg) \\
\hline Injector Mott & $72.21 \pm 0.24$ & $88.79 \pm 0.34$ \\
Hall A Compton & $72.67 \pm 1.01$ & $-175.79 \pm 0.75$ \\
Hall A Møller & $76.92 \pm 1.11$ & $-176.12 \pm 0.62$ \\
Hall B Møller & $69.71 \pm 0.63$ & $60.41 \pm 0.51$ \\
Hall C Møller & $73.24 \pm 0.54$ & $-56.95 \pm 0.63$ \\
\hline
\end{tabular}

certainties of the polarimeters the relative analyzing power between the five polarimeters is shown in Fig. 5.

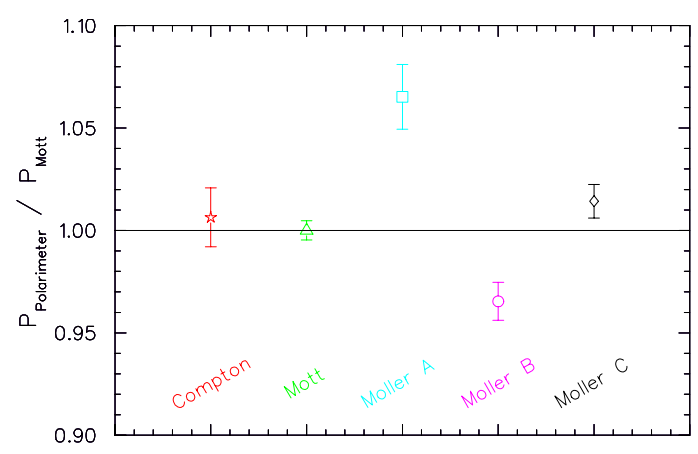

Figure 5: Relative analyzing power of the five electron beam polarimeters normalized to the Mott for comparison.

\subsection{Beam Energy Measured by Spin Precession}

The final beam energy can be directly measured from the precession of the beam polarization in the accelerator. As the electron beam gains energy in linac sections and transports through recirculation and experimental hall transport magnets the polarization precesses about the magnetic fields encountered. Two methods using spin precession to extract the final beam energy are presented.

The first method uses the precession difference $(\Delta \Psi)$ between the injector Mott and an experimental hall polarimeter. The final beam energy is,

$$
E=\frac{\frac{2 m_{e} \Delta \Psi}{a}-E_{0}\left(\theta_{t}-\theta_{h}\right)-\frac{5 E_{12}\left[\theta_{t}-\theta_{h}+4 \theta_{12}\right]}{9}}{\theta_{t}+\theta_{h}},
$$

where $E_{0}$ is the injector energy, $E_{12}$ is the inequality of the first and second linacs, $\theta_{t}$ is the total bend angle experienced by the beam, $\theta_{12}$ is the angular skewness of the two linacs, and $\theta_{h}(h \in A, B, C)$ is the bend angle into each respective end-station after the beam switchyard. For $\left|E_{12}\right|<10 \mathrm{MeV}$ (1.8\% linac variation) $\frac{\Delta E}{E} \sim 10^{-5}$. This level of linac equality is acheivable and makes such a high precision energy measurement feasible.

The second method uses only the part of the total precession which results from separating the beams to different end-station polarimeters at fixed energy after the beam switchyard. The advantage is the uncertainties in the injector energy, linac equality, and arc bend angles are eliminated. The beam energy calculated using two end-station polarimeters is simply,

$$
E=\frac{m_{e} c^{2}}{a} \cdot \frac{\Delta \Psi}{\Delta \Theta}
$$

where $\Delta \Psi$ and $\Delta \Theta$ are the measured precession and bend angle between the two polarimeters. In this case the uncertainty in bend angle is the main contribution. The disadvantage of this method is the precession between end-station polarimeters is much smaller than of the entire accelerator. Even at the maximum JLab energies $\frac{\Delta E}{E} \sim 10^{-3}$.

A third comparative method, used the Hall $\mathrm{A}$ arc as a spectrometer to determine the beam energy. Two pairs of high precision beam profile monitors measured the beam deflection through 8 calibrated arc dipole magnets yielding a final beam energy of $\mathrm{E}=5646.5 \pm 3.0 \mathrm{MeV}$.

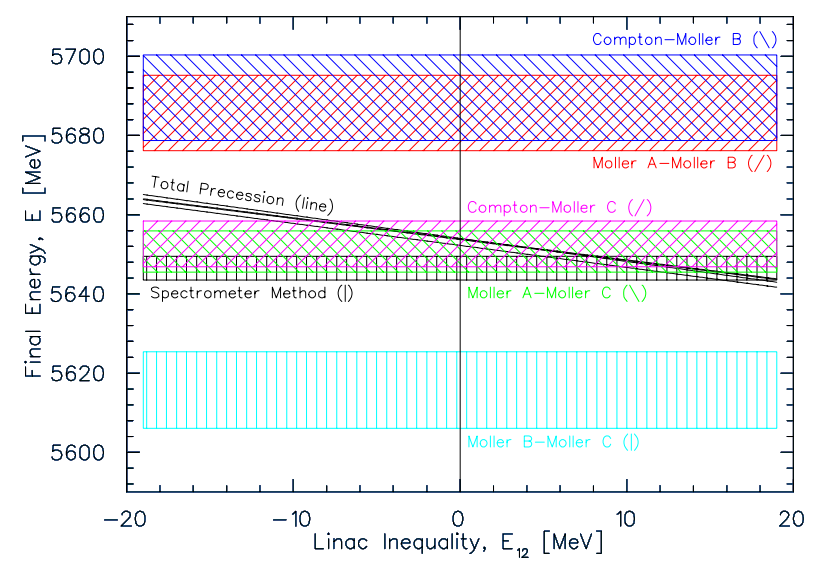

Figure 6: All energy results for $\left|E_{12}\right|<20 \mathrm{MeV}$.

A summary of the ten energy measurements is displayed in Fig. 6. A central value near $5648 \mathrm{MeV}$ was measured with a variation of $1.3 \%$. The sensitivity of the spin precession to arc bend angle reveals a discrepancy of $\pm 35 \mathrm{MeV}$ whose sign is correlated to whether the Hall B polarimeter is compared to Hall $\mathrm{A}$ or $\mathrm{C}$ polarimeters. A possible explanation is that the Hall $\mathrm{B}$ beamline is $-0.22^{\circ}$ off center relative to design.

\section{CONCLUSIONS}

The first comparison of five different electron polarimeters of three types (Mott, Compton, Møller) was made. JLab has the resources to facilitate high precision electron polarimetry $\left(\frac{\Delta P}{P}<1 \%\right)$, however, understanding the instrumental uncertainties of the polarimeters is clearly required.

Seven of the ten energy measurements (excluding the Hall B Møller results) by two independent spin precession and one spectrometer methods agree with variation $<0.4 \%$ (for $\left|E_{12}\right|<10 \mathrm{MeV}$ ). In this region $\frac{\Delta E}{E} \sim 10^{-5}$ for the total spin precession method.

I acknowledge the support of the JLab polarimeter groups and Accelerator Division staff, numbering more than 40 , who made the experiment possible. 